\title{
Development and validation of a novel quality control methodology on screening out for human osteosarcoma cell aptamers
}

Hua Wang

Beihua University

Ji Liang ( $\nabla$ yanshuang_bhdx@163.com )

Guilin Medical Universtiy https://orcid.org/0000-0003-2640-4305

Yan Ma

Beihua University

Lei Zhou

Beihua University

Yufei Zhang

Guilin Medical University

Mingcheng Li

Beihua University

\section{Research Article}

Keywords: Human osteosarcoma, Aptamer, Methodology, Spectrofluorimetry, Flowcytometry

Posted Date: February 19th, 2021

DOl: https://doi.org/10.21203/rs.3.rs-175435/v1

License: (c) (i) This work is licensed under a Creative Commons Attribution 4.0 International License. Read Full License 


\section{Abstract \\ Objectives}

To establish an accurate, time-saving quality-monitoring method in screening for tumour cell aptamers in order to shorten the screening process and ensure the accurate preparation of the aptamer.

\section{Results}

During quantitative PCR for U2-OS and HOS template, the results showed that the bands obtained from 14 cycles were bright and no non-specific amplification within the optimal template concentrations between 19.0 and $21.0 \mathrm{ng} / \mu \mathrm{l}$. Each round of forward screening was accompanied by reverse screening to accelerate the elimination of non-specific single-strand DNA (ssDNA). In the meanwhile, the aptamer groups were effectively purified specifically bounding to target cells. Besides, we observed that the fluorescence spectroscopy is more accurate, time-saving, and convenient for quality control compared with flow cytometry.

\section{Conclusion}

The method proposed in the study is appropriate for the rapid screening out for human osteosarcoma cell aptamers. The quantitative template concentration, forward screening with back screening, and fluorescence spectroscopy are important methods for accurate preparation and quality control of tumour cell aptamers. It can provide scientific reference data for the amplification of dsDNAs in other sublibraries.

\section{Introduction}

Osteosarcoma (OS) is the most common malignant bone cancer, which occurs in $5 \%$ of children and adolescents (Yang et al. 2013). The main treatments for OS include surgery and chemotherapy, however, the overall survival of patients with metastatic OS remains unsatisfactory at less than $30 \%$ पYan et al. 2018区. Increasing evidence has demonstrated that advances in the diagnosis and treatment of OS can improve the clinical outcome in OS patients [Wang et al. 2003]. Therefore, it is vital to develop novel molecular biomarkers for diagnosis and prediction of OS that can predict clinical prognosis and outcomes and improve OS therapeutic strategies.

Aptamers are single-stranded DNA or RNA oligonucleotides that selectively bind to various targets ranging from proteins, amino acids, metal ions to viruses or bacteria (Nimjee et al. 2017; Adachi et al. 2019). Thereafter, the oligonucleotides were used as templates to be amplified and screened to confirm their sequences by PCR. This repeated combination, amplification, and screening process finally enriches oligonucleotides with high affinity. Theoretically, a single-stranded oligonucleotide can spontaneously curl 
into a three-dimensional spatial structure, identify the spatial structure of the target molecules, and combined with each other by hydrogen bonds, ionic bonds and van der Waals forces, has high specificity and high affinity for antigen-antibody reactions $\square$ Chen et al. 2015区.

A high-flux oligonucleotide library contains all possible stereostructures, so it is possible to screen high affinity ligands of any kind of target molecules therefrom. Changes in cell membrane proteins or other substances caused by loss, insertion or point mutation of certain genes in tumour cells give rise to differences in membranes epitope complexity between tumour cells and normal cells, which inspire people to use aptamers instead of antibodies as diagnostic probes for clinical tumours or as targeting elements for tumour therapy (Takahashi et al. 2018). Compared with antibodies, aptamers not only exhibit specific recognition and binding, but also have the following unique features: low molecular weight, low immunogenicity, easy chemical synthesis and label modification, easy transportation, and can survive long-term storage and application. These bestow incomparable advantages over traditional chemical and immunological methods: it has been applied to the research on the diagnosis and treatment of clinical tumors with aptamer biosensors, and also allows biosensors to be used in the detection of clinical microbial infections, food science, etc\Del et al.2011; Shamah et al. 2008区.

Evidence proved that the ideal target element required precise monitoring technology in the preparation process to ensure enough specificity and affinity between the screened aptamer and the target. This is the key challenge to establish the aptamer probe and biosensor and is also the premise for the application and development of such a biosensor in clinical diagnosis and treatment in the future (Haßel et al. 2019; Yu et al. 2018).

Based on cell-SELEX screening technology, we have successfully developed a novel molecular aptamer for recognition of human osteosarcoma cell using the cell-SELEX method, u2-OS and HOS cell lines were selected as target cells for aptamer screening (Wang et al. 2015). However, we found that the quality monitoring become a key, resulting in success or failure in the screening process. One was implementation of template amounts in amplification of dsDNA by PCR. Generally, template in volume percentage is intended to be the inaccuracy of the parameters. Besides that, the operator repeated optimisation of the experimental process and would bring about a loss of many secondary library ssDNA. In the meanwhile, flow cytometry was mainly used for affinity identification of secondary libraries in previous reports in the selection process of aptamer, it is complicated, time-consuming and cannot be popularised and used in routine analytical laboratories as a classic and commonly used method.

In this study, we managed to develop a novel quality control methodology on screening out for human osteosarcoma cell aptamers. Human fibrosarcoma cells were used as reverse screening cells of OS cells to obtain a high-fidelity sub-library, and reverse screening was conducted to track each round of forward screening process. We implemented of the quantitative template instead of volume percentage, thus accelerating the elimination of non-specific or less specific ssDNAs and realise the purpose of effectively purifying the screened sub-library. Additionally, the fluorescence spectroscopy was applied to track the 
affinity of secondary library, expecting to obtain a monitoring method that is simple, accurate, timesaving, simple and easy to operate, which can be popularised in routine experimental work.

\section{Materials And Methods}

\section{Materials}

The 76nt random oligonucleotide library, 5'-ATC CAG AGT GAC GCA GCA-40nt-TGG ACA CGG TGG CTT AGT-3',FITC labelled upstream primer 5'FITC-ATC CAG AGT GAC GCA GCA, and Biotin labelled downstream primer 5'Biotin-ACT AAG CCA CCG TGT CCA-3' were synthesised by Huada Biotech (Beijing, China) Co., Ltd. U2-OS cells, HOS cells, and HT-1080 cells were purchased from Boshide Bioengineering Co., Ltd. McCoy's 5A medium and high-sugar DMEM medium were all purchased from Boshide Bioengineering Co., Ltd (Wuhan, China). Yeast tRNA (Fluka Analytical, USA), heat-inactivated foetal bovine serum (FBS Invitrogen, USA), Streptavidin Sepharose ${ }^{\circledR}$ HP (GE healthcare, USA), RPMI-1640 medium (GIBCO), 2x Taq PCR Master Mix, 50 bp DNA Ladder (Tiangen, Japan), and an agarose gel recovery kit (Dingguo, China) were also used.

Cell culture and sub-library construction

McCoy's 5A and high-sugar DMEM containing 10\% foetal bovine serum were employed to culture U2-OS, HOS and HT-1080 tumour cells respectively. Cells with an activity of more than $95 \%$ were prepared 24 hours before the experiment, and the cell count was $0.5 \times 10^{6} \mathrm{cells} /(100 \mathrm{~mm} \times 20 \mathrm{~mm})$ in a Petri dish (cultured overnight). The initial oligonucleotide library 5'-ATC CAG AGT GAC GCA GCA-40nt-TGG ACA CGG TGG CTT AGT-3' was dissolved in $1000 \mu$ of binding buffer (the final concentration of ssDNA was $10 \mathrm{nM}$ for the first binding reaction and $1000 \mathrm{nM}$ for the second binding reaction), denatured at $95{ }^{\circ} \mathrm{C}$ for 5 minutes, and placed in ice water for later use.

After the cells were thrice-washed with rinsing buffer, the residual liquid was drained, and the precooled binding buffer containing the candidate library was added to the cell culture plate for binding reaction at $4^{\circ} \mathrm{C}$ and $50 \mathrm{rpm}$. It took one hour for one to four rounds, and the reaction time was shortened to 30 minutes after four rounds. After the reaction was terminated, the cells were washed with rinsing buffer to remove ssDNAs that did not bind to the cells. Cells were harvested with a cell shovel into a 1.5-ml centrifuge tube, heated at $95^{\circ} \mathrm{C}$ for $10 \mathrm{~min}$ to separate ssDNAs bound to the cell surface from the cells, centrifuged at $13,000 \mathrm{rpm}$ at $4^{\circ} \mathrm{C}$ for $5 \mathrm{~min}$, and the supernatant was collected. SsDNAs in the supernatant were used as a template, double-stranded oligonucleotides (dsDNAs) were prepared in large quantities by PCR. Streptavidin Sepharose affinity chromatography columns were used to dissociate double strands, and the harvested positive-stranded ssDNAs would be used as an aptamer sub-library for the next round of screening.

Optimisation of template quantitative concentration and amplification cycle times 
Each round of ssDNAs dissociated from the cell surface needs PCR amplification to generate enough dsDNA to obtain enough ssDNAs for the next round of aptamer screening. The optimisation of PCR amplification system and parameters is a necessary step. Previous literature reported that the template concentration in the PCR reaction system was basically optimised by percent volume (Su et al. 2018; Tan et al. 2016). For example, in a 100- $\mu$ PCR reaction system, $50 \mu \mathrm{l}$ of 2x Taq PCR Master Mix was included, the final concentration of upstream and downstream primers was $0.5 \mu \mathrm{M}, 10 \mu \mathrm{l} \%, 20 \mu \mathrm{l} \%$, and $40 \mu \mathrm{l} \%$ of template ssDNAs were respectively placed in three reaction tubes, and $\mathrm{dd}_{2} \mathrm{O}$ supplemented the reaction volume to $100 \mu \mathrm{L}$. In this study, quantitative template concentration was adopted to optimise the cycle times simultaneously: U2-OS ssDNA concentration was measured at $139 \mathrm{ng} / \mu \mathrm{l}$, and $27.8 \mathrm{ng} / \mu \mathrm{l}: 13.9 \mathrm{ng} / \mu \mathrm{l}$ and $6.95 \mathrm{ng} / \mu \mathrm{l}$ were obtained after 5 -fold, 10 -fold, and 20 -fold dilution. The $12.5-\mu \mathrm{l}$ amplification system contained $6.25 \mu \mathrm{l}$ of $2 \mathrm{x}$ Taq PCR Master Mix, $0.5 \mu \mathrm{l}$ of upstream primer, and $0.5 \mu \mathrm{l}$ of downstream primer (the final concentration was $0.5 \mu \mathrm{M}$ ), $3 \mu \mathrm{l}$ of each concentration template, and $2.25 \mu \mathrm{l}$ of $\mathrm{ddH}_{2} \mathrm{O}$. PCR temperature parameters: denaturation temperature $95^{\circ} \mathrm{C}$ for $30 \mathrm{~s}$, annealing temperature $56.3^{\circ} \mathrm{C}$, followed by extension at $72{ }^{\circ} \mathrm{C}$ for $30 \mathrm{~s}$. PCR systems with each template concentration were subjected to $8,10,12$, and 14 cycles respectively. The results showed that the $6.95 \mathrm{ng} / \mu \mathrm{l} \times 3 \mu \mathrm{l}$ template and the bands obtained in 14 cycles were bright and non-specific amplifications $3 \%$ agarose gel electrophoresis. The template quantitative experiment of HOS cells was similar to that U2-OS. Whereas, PCR in volume template often exhibited unsatisfactory optimisation results, requiring redesign of template volume, cycle number, and even ion and temperature parameters.

Comparison on affinity monitoring of sub-libraries by fluorescence spectroscopy and flow cytometry

Starting from the sixth round, the screened sub-library ssDNAs were analysed by flow cytometry and fluorescence spectrum images, and subsequent experiments such as whether the screening process was terminated or not, and continuing cloning and sequencing, were determined according to image drift. For flow cytometry monitoring: 5'-FITC-ssDNA was synthesised by the company, or 5'-FITC-forward primer and 5'-Biotin-reverse primer were used to prepare double-labelled products by PCR, and then 5'-FITCssDNAs were prepared through alkaline lysis affinity chromatographic column separation. 5'-FITCssDNAs obtained by alkaline lysis needed desalting and purification for later use. The reaction process

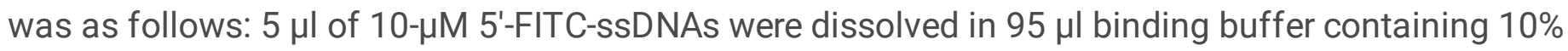
bovine serum albumin (the final concentration was $500 \mathrm{nM}$ ), meanwhile, $500 \mathrm{nM}$ unlabelled ssDNA was prepared for blank control. Each target cell used in the experiment was rinsed three times with $0.5 \times 10^{6}$ rinse buffer and dissolved in $100 \mu \mathrm{l}$ binding buffer (containing 10\% FBS); $50 \mu \mathrm{l}$ of cells and $50 \mu \mathrm{l}$ of standby ssDNA were gently mixed, and the binding reaction was conducted at $4^{\circ} \mathrm{C}$ and $50 \mathrm{rpm}$ for 30 minutes. After rinsing the cells, they were dissolved in $200 \mu \mathrm{l}$ of binding buffer for flow cytometry analysis.

Fluorescence spectrum analysis: the target cells were cultured by counting $0.5 \times 10^{6}$ cells from 24 hours ago, rinsing three times with the rinsing solution in the next day, then the cells were dissolved in the binding buffer solution containing the final concentration of ssDNAs of $1000 \mathrm{nM}$, and the cells were rinsed after binding reaction at $4^{\circ} \mathrm{C}$ and $50 \mathrm{rpm}$ for 30 minutes to remove unbound ssDNAs. The cells 
were then dissolved in $300 \mu \mathrm{l}$ of binding buffer at $95^{\circ} \mathrm{C}$ for 10 minutes: ssDNAs bound to the cell membrane surface were separated from the cells, centrifuged at 13,000 rpm for 5 minutes, and the supernatant harvested. $150 \mu \mathrm{l}$ of the supernatant was used as a template, and PCR amplification was performed using double labelled primers. $200 \mu \mathrm{l}$ of Streptavidin Sepharose was loaded into the chromatography column, DPBS fully rinsed gel particles in the column, then $300 \mu$ l of double-labelled dsDNA from PCR amplification was added, and the chromatography column was fully rinsed with DPBS with at least three column bed volumes. $200 \mathrm{mM} \mathrm{NaOH}(500 \mu \mathrm{l})$ was added for alkaline lysis, and alkaline lysis solution containing 5'FITC-ssDNA-3' of the positive chain was collected. Fluorescence spectrum was used to monitor the lysis solution at an excitation wavelength of $492 \mathrm{~nm}$ and emission wavelength of 520 $\mathrm{nm}$.

Reverse screening of sub-libraries of U2-OS and HOS Cells by HT-1080 Cells

Clinical diagnosis of OS needs to be differentiated from fibrosarcoma and chondrosarcoma. Although their affinity has not yet been revealed, the characteristics of inducement, infiltration and metastasis imply that there are similarities between cell epitopes of OS cells and fibrosarcoma cells, so fibrosarcoma cells were selected as reverse screening cells. The reverse screening process was slightly different from the forward screening of target cells: each round of forward screening sub-library ssDNAs was dissolved in $700 \mu$ lo $1000 \mu$ l binding buffer (final concentration $1000 \mathrm{nM}$ ), heated to $95^{\circ} \mathrm{C}$ for denaturation for 5 minutes, and then placed in ice water for later use. High-sugar DMEM containing $10 \%$ FBS cultured HT1080 tumour cells was used: cells with an activity of more than $95 \%$ were prepared 24 hours before the experiment, and the cell count was $0.5 \times 10^{6}$ cells / (100 mm $\times 20 \mathrm{~mm}$ Petri dish) (cultured overnight). After the cells were thrice-washed with rinsing buffer, the residual liquid was drained, and the precooled binding buffer containing library SsDNAs was added to the cell culture plate, and the binding reaction was conducted for $45 \mathrm{~min}$ at $4^{\circ} \mathrm{C}$ and $50 \mathrm{rpm}$. SsDNAs that had not been bound to HT-1080 cells remained in the binding buffer, so after the reaction was terminated, the liquid in the Petri dish was harvested into a $1.5-\mathrm{ml}$ centrifuge tube, and the OD value was determined. DsDNAs and Streptavidin Sepharose affinity chromatographic columns were prepared in large quantities by PCR according to the above method to dissociate double strands, and the harvested positive strand ssDNAs were purified, whereafter specimens were stored or used for the next round of screening of positive aptamers.

\section{Results}

Optimisation of template concentration quantification and number of amplification cycles

The U2-OS and HOS cells SSDNA were used to conduct experimental template quantification and optimisation of amplification cycle times as well as volume template optimisation. The results revealed the PCR amplication of the two tumour cells ssDNAs with different quantification concentrations and different cycle times: in the 12.5- $\mu$ l amplification system, the template amount can obtain dsDNAs bands with ideal purity and amplification amount in 19.0 to $21.0 \mathrm{ng} / \mu \mathrm{l}$ and 14 cycles, suggesting that a one-time 
optimisation experiment can obtain many parameters for preparing a large amount of dsDNA, providing information that can be followed for subsequent rounds of library screening (Fig. 1a and 1b).

Additionally, the findings showed that U2-OS ssDNAs with undetermined concentration resulted in PCR amplification results of a percent volume template: the $10 \%$ volume template exhibited slightly clear bands after 10 or 12 cycles, but the amplification products were too few to be used. The 10 circular bands of $40 \%$ volume template were relatively bright, indicating that the amplification was acceptable, but there was non-specific amplification at the top and bottom (Fig. 1C), therefore, the template volume, number of PCR cycles, and even other temperature parameters needed to be redesigned, resulting in the repeated operation losing a large number of ssDNA templates and prolonging the experimental process.

Comparison on affinity monitoring of sub-library by fluorescence spectroscopy and flow cytometry

U2-OS and HOS cells were used as target cells, flow cytometry and fluorescence spectrum image analysis were applied to the screened sub-library ssDNAs from the sixth round, and whether the screening process was terminated or not, cloning sequencing and other subsequent experiments were determined according to image drift. The fluorescence spectrum monitoring results demonstrated that the fluorescence curves of the sub-libraries of the two cells had no obvious drift in the ninth and tenth rounds, and the overlapping curves were clearly visible, indicating that the specificity and affinity of the sub-library ssDNAs obtained in the ninth and tenth rounds to the target cells tend to be stable, indicating the node of the aptamer screening process (Fig. 2 and Fig. 3). The flow cytometer monitoring results suggested the effective identification of specificity and affinity tendency of U2-OS ssDNA sub-library by flow cytometry: the green peak of SsDNAs of the simple library was located on the left-hand side of the horizontal axis of coordinates as a control while cells specifically bind with ssDNAs, the complexity of the measurement system increases, resulting in the right-shift of the sample peak (Fig. 4). The results showed that the peaks of each round of cells-ssDNAs shifted to the right were superimposed, which was not as easy to judge as the data shown by the fluorescence spectrum.

HT-1080 cells in the reverse screening of sub-library of U2-OS and HOS cells

In the second round, U2-OS and HOS cells began to track reverse screening after obtaining sub-libraries. Implementation of forward and reverse screening was performed in parallel. After 40-fold dilution of the forward sub-library ssDNA sample, fluorescence spectrometry was performed. Results showed that the spectral curves of the ninth and tenth rounds overlap and were clearly separated from the spectral curves of other rounds. The test results of diluted samples further confirmed that the accompanying reverse screening effectively accelerated the elimination of non-specific ssDNAs (Fig. 5 and Fig. 6). The superposition of curves from the ninth and tenth rounds illustrated that the specificity and affinity of the two rounds of sub-libraries and target cells tended to be coherent, suggesting that the sub-libraries obtained from the ninth round of screening can be used for subsequent cloning and sequencing.

\section{Discussion}


At present, oligonucleotide aptamers as ideal target recognition elements are leading biosensors to be used in clinical accurate diagnosis and treatment. Ensuring sufficient specificity and affinity between the screened aptamer and the target is a key challenge for establishing aptamer disease diagnosis probes and biosensors and is a prerequisite for the development of biosensors in future clinical diagnosis and treatment (Sá et al. 2013; Sefah et al. 2010). How to prepare excellent aptamers is a topic worth thinking about and practicing.

In the present study, we started with the process of preparing dsDNAs by PCR, many dsDNAs need to be prepared owning to each round of screening. Before mass preparation, the template, number of cycles, temperature and related ion concentration required for PCR amplification required to be selected and determined through optimisation experiments. We found that optimisation of template concentration in volume percent often needed to be repeated while other conditions were determined, and many samples were lost. The missing sample may contain the oligonucleotides most specific to the target cell, so the ssDNA library prepared was imperfect, therefore, the template concentration was quantitatively explored whereas other parameters 3454 determined, the four gradient concentrations of the template were amplified by four cycles, respectively. As a result, more accurate template concentration data and number of cycles can be acquired by one-time experiment, which provided scientific quantitative indices for subsequent dsDNAs preparation of sub-libraries, while avoiding the loss of oligonucleotide sequences. Using exact template concentration for amplification was our first and successful attempt.

After the sixth round of aptamer screening, we began to detect the specificity of ssDNAs binding to cells in the secondary library. We adopted flow cytometry and fluorescence spectroscopy respectively. In the process of flow cytometric monitoring, ssDNAs need to be purified by using a kit, cloned, sequenced, and then the sequence was submitted to the company for labelling the aptamer probe. On the other hand, when the binding characteristics of the library were not monitored during the period, the secondary libraries of rounds 6 to 13 can only be intensively purified after 12 to 13 rounds, and the cloning sequencing and the submission of sequence synthesis marker probes can be completed at the same time. The process was complex, long and expensive. If ourselfies prepared fluorescent labelled ssDNA probe, it needs purification before binding reaction with cells, and the screening time of each round was also prolonged. In this study, fluorescence spectroscopy was used to conveniently track the affinity monitoring of each round of screened sub-library ssDNAs without prior cloning and sequencing. Our findings showed that SSDNA fluorescence spectra with similar affinity to tumour cells were clearly superimposed and easy to judge. In addition, the instruments used for fluorescence spectrum detection were easy to be operated and can be popularised for use in routine laboratory practice. Flow cytometric analysis observes the tendency of curve displacement to the right. When two or three curves drift to a less obvious extent (or overlap), it indicated that the screening can be finished; however, it is difficult to judge due to the smaller curve displacement, so it is necessary to increase the number of screening rounds, thus prolonging the experiment.

OS has strong invasiveness and metastasis (Wolter et al. 2017). The inducing factors include trauma and stimulation, viral infection, exposure to radioactive substances, and Rb gene abnormalities $\square$ Goto et al. 
2017】. It is necessary to differentiate fibrosarcoma, especially bone fibrosarcoma in the process of clinical diagnosis [Bayat et al. 2018]. Osteomyelitis fistula, sinus tract, and radiotherapy are factors inducing fibrosarcoma. It has been found that $91-100 \%$ of infantile fibrosarcoma has gene NTRK fusion, which indicates that there are congenital genetic factors $\square$ Ottaviani et al. 2009; Shamah et al. 2008区. Although their epigenetics have not been revealed at present, the characteristics of inducement, infiltration, and metastasis show that the cell epitopes of OS cells and fibrosarcoma cells are similar, so fibrosarcoma cells are selected as reverse screening cells $\square$ Sefah et al. 2010; Tang et al. 2007凶. Using similar tumour cells as reverse screening cells plays a more effective purification role. Our findings indicated that the fluorescence curves of the ninth and tenth rounds show ideal overlap, showing the similarity of purity and affinity of the oligonucleotide library.

We have also limitations of this study. The numbers of human u2-OS and HOS cell lines included in the study were not sufficiently enough to conclusively determine the specific and predictive power of this aptamer family. Further large-scale validation studies by patient-derived OS are needed to explore their value as predictive biomarkers for OS.

In summary, the method proposed in the study is appropriate for the rapid screening out for human osteosarcoma cell adaptor. The quantitative template concentration, forward screening with back screening, and fluorescence spectroscopy are important methods for accurate preparation and quality control of tumour cell aptamers. This study provides an easy-to-implement quality control method for high-quality preparation of aptamers.

\section{Declarations}

\section{Acknowledgements}

We thank the Professor Guilian Fu for an excellent review of our manuscript.

Disclosure of conflicts of interest The authors declare that they have no competing interests. The authors alone are responsible for the content and writing of the paper.

\section{References}

Adachi T, Nakamura Y (2019) Aptamers: A review of their chemical properties and modifications for therapeutic application. Molecules 24(23):4229.

Bayat P, Nosrati R, Alibolandi M, et al (2018) SELEX methods on the road to protein targeting with nucleic acid aptamers. Biochimie 154:132-155.

Chen YU, Xu SF, Xu M et al (2015) Postoperative infection and survival in osteosarcoma patients: Reconsideration of immunotherapy for osteosarcoma. Mol Clin Oncol 3(3):495-500. 
Del M S, Kurek KC, Stein GS et al (2011) Role of the WWOX tumor suppressor gene in bone homeostasis and the pathogenesis of osteosarcoma. Am J Cancer Res 1(5):585-94.

Haßel SK, Mayer G (2019) Aptamers as therapeutic agents: Has the initial euphoria subsided? Mol Diagn Ther 23(3):301-309.

Goto S, Tsukakoshi K, Ikebukuro K (2017) Development of aptamers against unpurified proteins. Biotechnol Bioeng 114(12):2706-2716.

Nimjee SM, White RR, Becker RC et al (2017) Aptamers as therapeutics. Annu Rev Pharmacol Toxicol 57:61-79.

Ottaviani G, Jaffe N (2009) The epidemiology of osteosarcoma. Cancer Treat Res 152: 3-13.

Su Y, Shao C, Huang X『et al (2018) Extraction and detection of bisphenol A in human serum and urine by aptamer-functionalized magnetic nanoparticles. Anal Bioanal Chem 410 (7): 1885-1891.

Sá LT, Simmons S, Missailidis S, et al. (2013) Aptamer- based nanoparticles for cancer targeting. J Drug Target 21(5): 427-34.

Sefah K, Shangguan D, Xiong X, et al (2012) Development of DNA aptamers using Cell-SELEX. Nature protocols 5(6): 1169.

Shamah SM, Healy JM, Cload ST (2008) Complex target SELEX. Acc Chem Res

41: 130-138.

Sefah K, Shangguan D, Xiong XL, et al (2010) Development of DNA aptamers using Cell-SELEX. Nature Protocols 5:1169-1185.

Tang ZW, Shangguan D, Wang K, et al (2007) Selection of aptamers for molecular recognition and characterization of cancer cells. Anal Chem 79: 4900-4907.

Takahashi M (2018) Aptamers targeting cell surface proteins. Biochimie 145:63-72.

Tan J, Yang N, Hu Z, et al (2016) Aptamer-functionalized fluorescent Silica nanoparticles for highly sensitive detection of leukemia cells. Nanoscale Rest Lett 11(1):298.

Wang C, Zhang M, Yang G et al (2003) Single-stranded DNA aptamers that bind differentiated but not parental cells: subtractive systematic evolution of ligands by exponential enrichment. J Biotech 102(1): 15-22.

Wang $\mathrm{H}$, Liang J, Ma Y, et al (2015) Identification of a novel molecular probe for recognition of human osteosarcoma cell using the cell-SELEX method. Int J Clin Exp Med 8(10):18151-18157. 
Wolter O, Mayer G (2017) Aptamers as valuable molecular tools in neurosciences. J Neurosci 37(10):2517-2523.

Yang J, Zhang W (2013) New molecular insights into osteosarcoma targeted therapy. Curr Opin Oncol 25: 398-406.

Yan AC, Levy M (2018) Aptamer-mediated delivery and cell-targeting aptamers: Room for rmprovement. Nucleic Acid Ther 28(3):194-199.

Yu S, Xu H, Chen Y (2018) Real-time and label-free detection of bisphenol A by an ssDNA aptamer sensor combined with dual polarization interferometry. New J Chem 42 (4), DOI: 10.1039/C7NJ05064J.

\section{Figures}
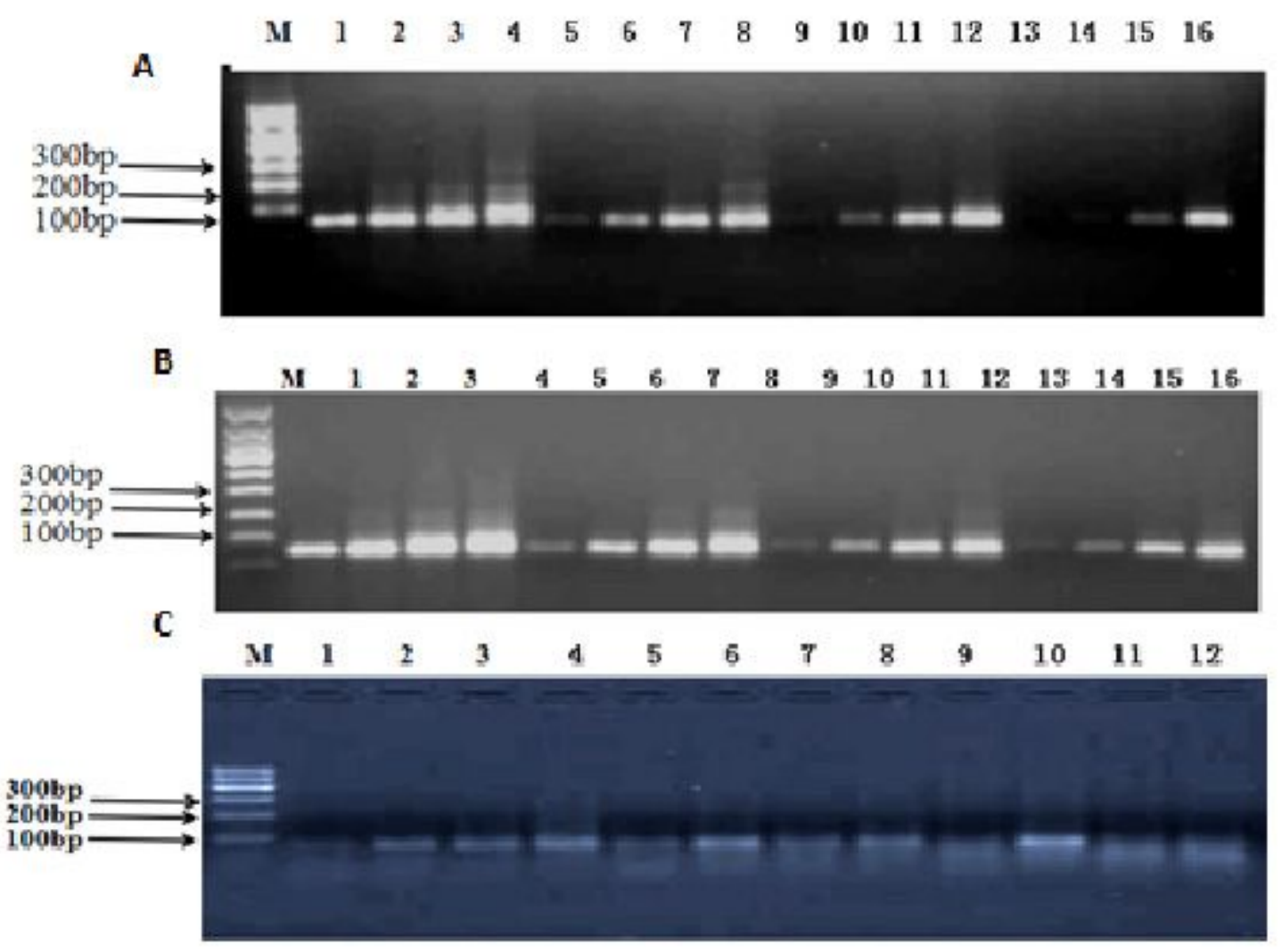

\section{Figure 1}

Agarose gel electrophoresis of optimization for template quantification and cycle times Note: A:

Optimization for quantification of template and cycles (ssDNAs fromU2-OS cells) M: DNA Marker; Lane 1区 4, lane $5 \llbracket 8$, lane $9 \varangle 12$ and lane $13 \varangle 16$ represented the templates' initial concentrations was $139 \mathrm{ng} / \mu \mathrm{l}$, the final concentrations was $27.8 \mathrm{ng} / \mu \mathrm{l}, 13.9 \mathrm{ng} / \mu \mathrm{l}$ and $6.95 \mathrm{ng} / \mu \mathrm{l}$ diluted in 5,10 and 20 times. The cycles were $8,12,14$ and18 respectively. B: Optimization for quantification of template and cycles (ssDNAs from

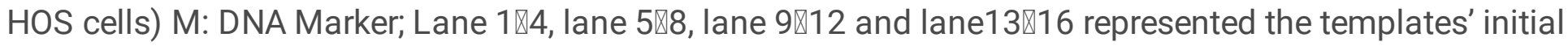


concentrations was $139 \mathrm{ng} / \mu \mathrm{l}$, the final concentrations was $27.8 \mathrm{ng} / \mu \mathrm{l}, 13.9 \mathrm{ng} / \mu \mathrm{l}$ and $6.95 \mathrm{ng} / \mu \mathrm{l}$ diluted in 5,10 and 20times. The cycles were $8,12,14$ and 18 respectively. C: Optimization for quantification of

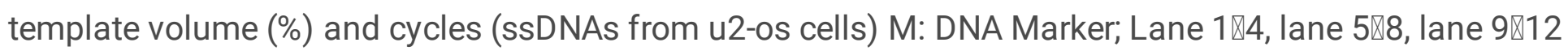
represented the templates' initial volume was $10 \%, 20 \%$ and $40 \%$. The cycles were $8,12,14$ and 18 respectively.

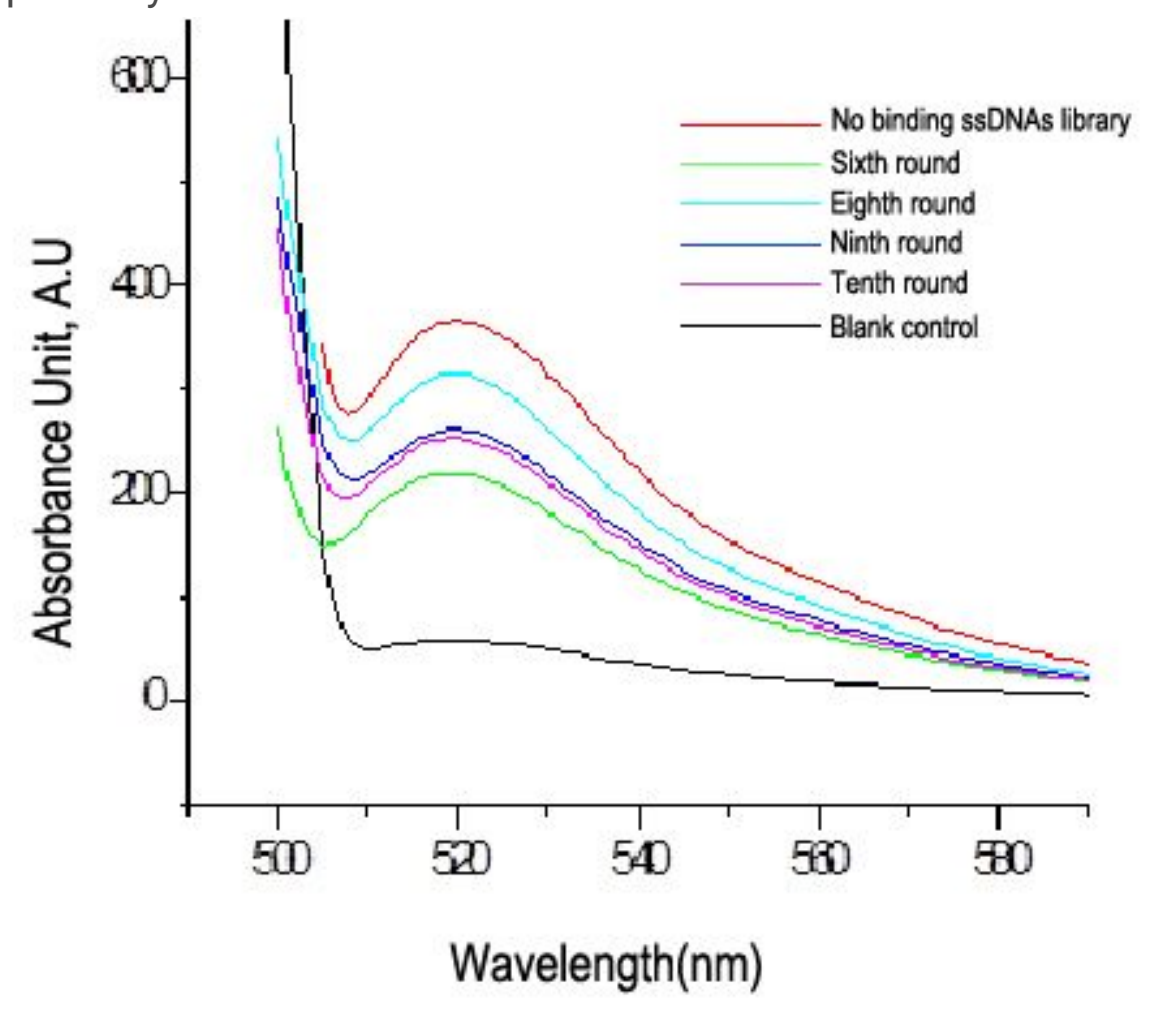

Figure 2

Fluorescence spectrum of sub-library of ssDNAs from U2-OS cells

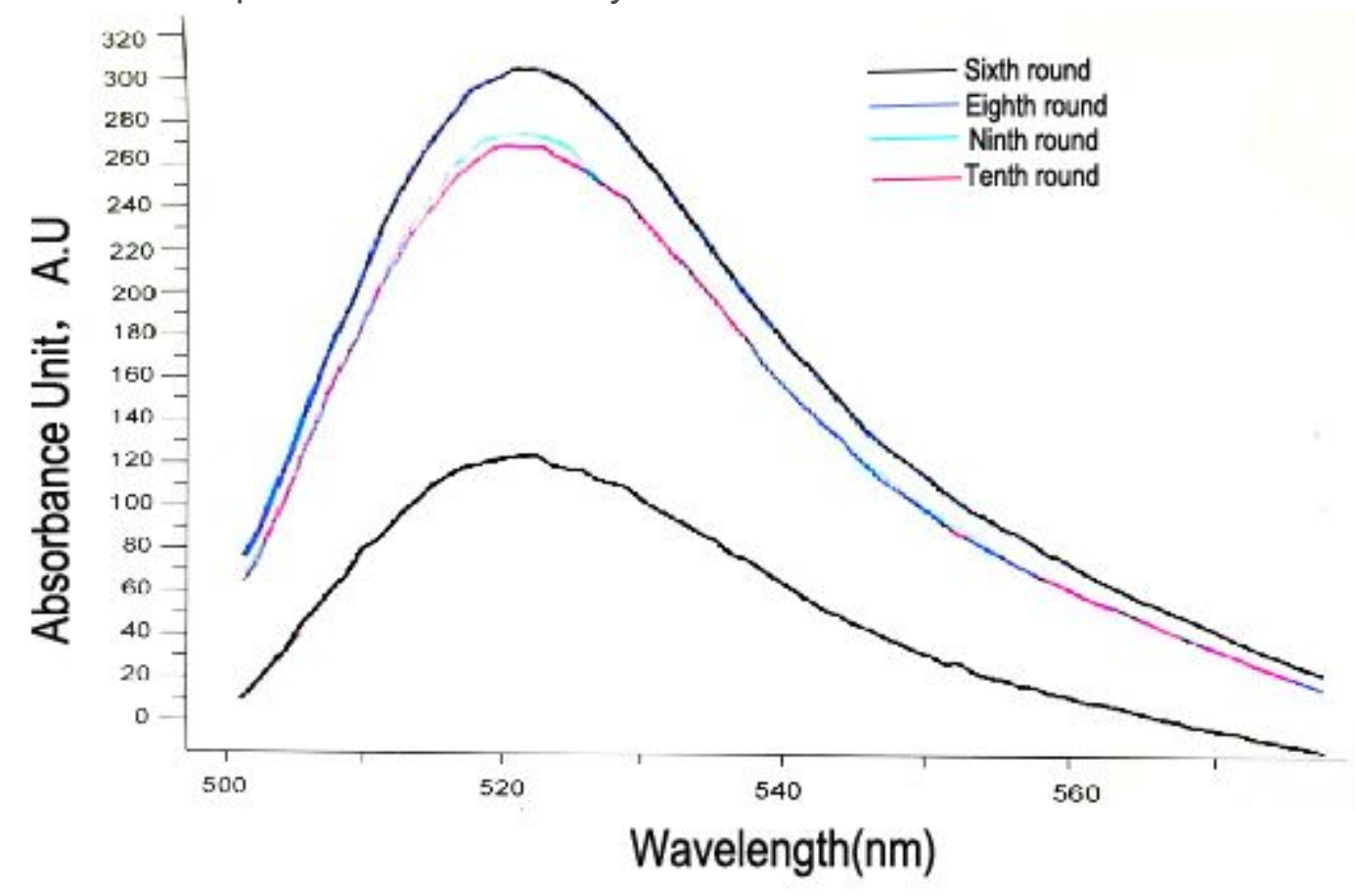


Figure 3

Fluorescence spectrum of sub-library of ssDNAs from HOS cells

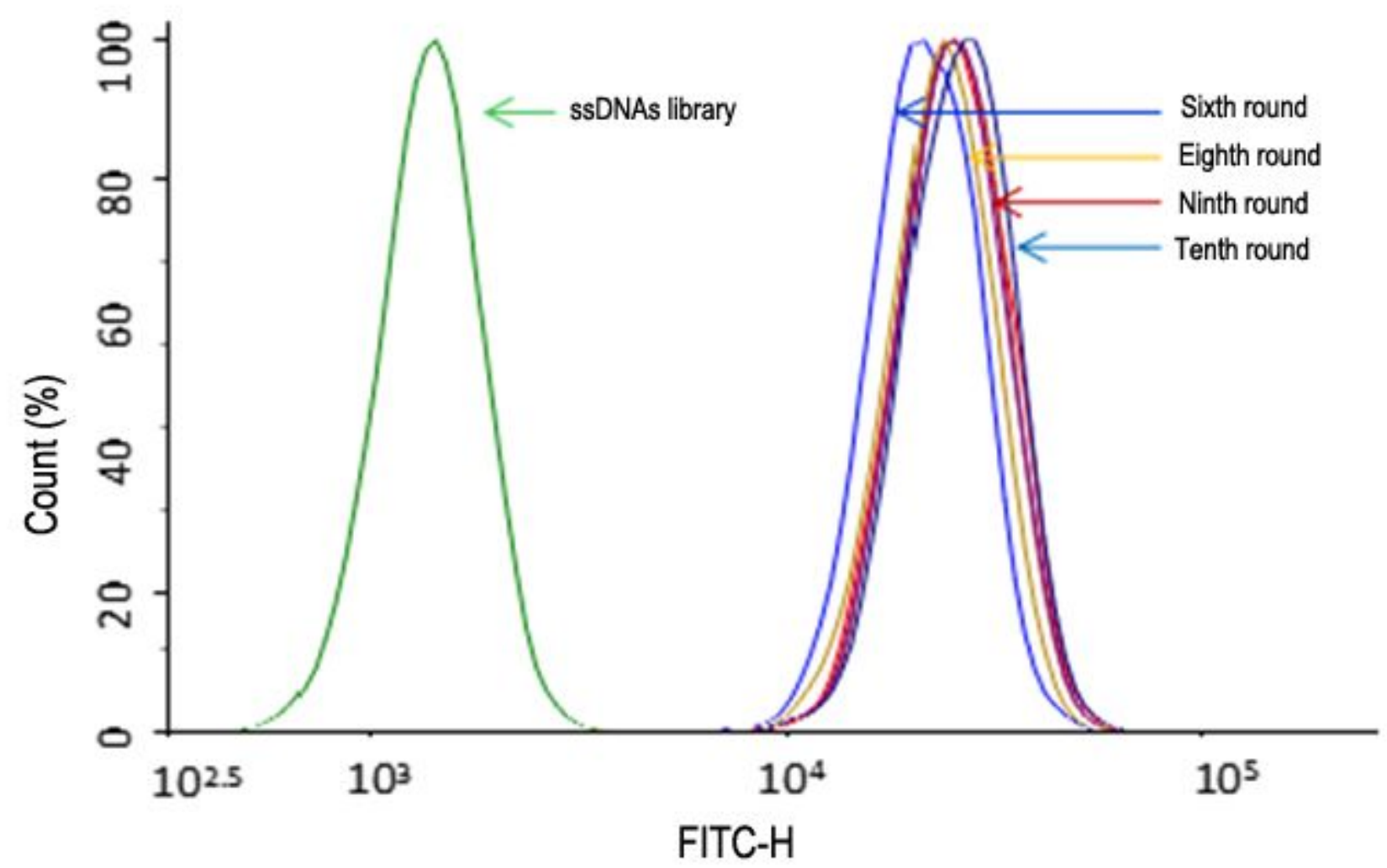

Figure 4

Flow cytometry monitoring of sub-library ssDNAs from U2-OS cells 


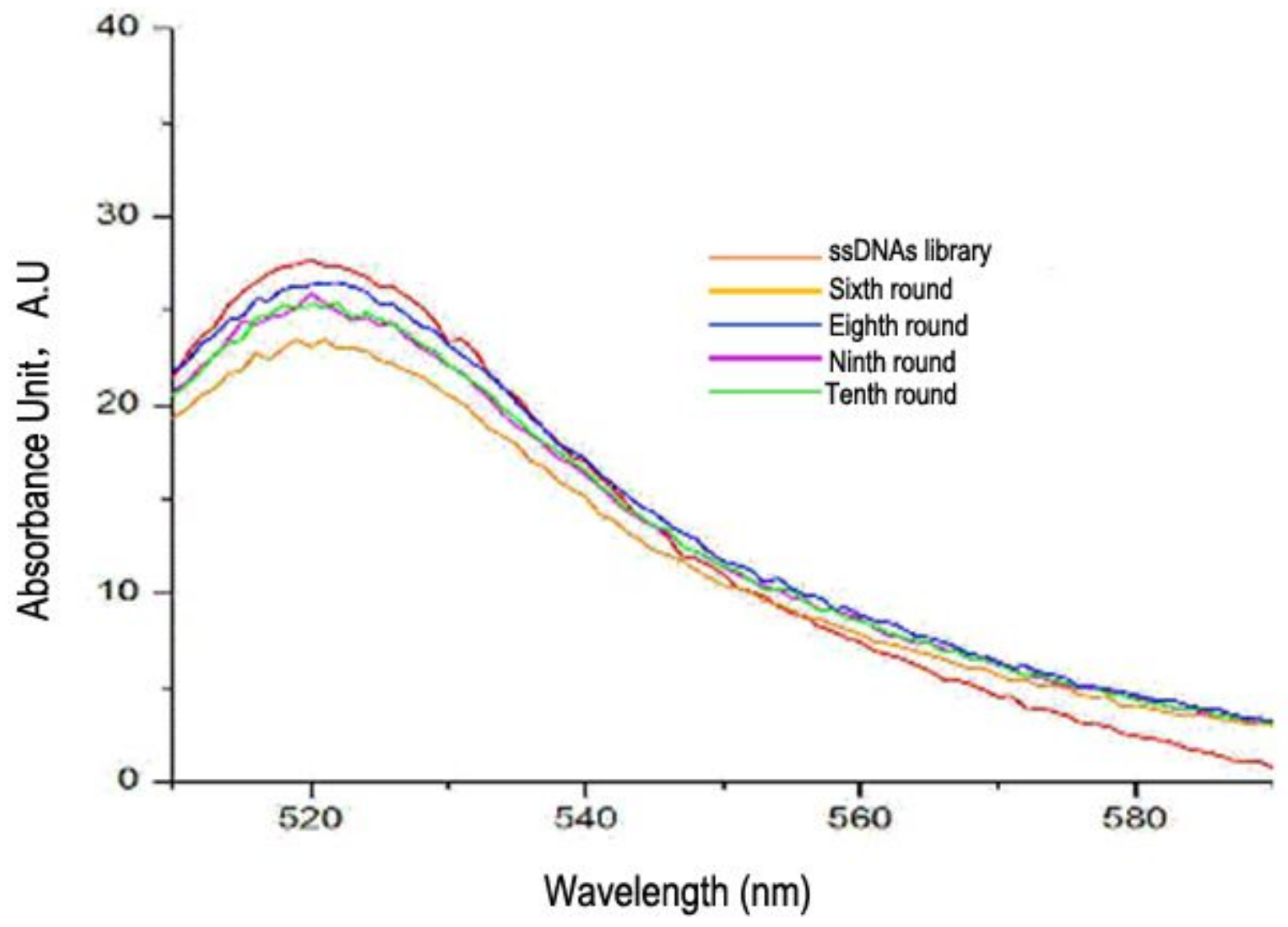

Figure 5

Fluorescence spectrum monitoring of U2-OS cell sub-library ssDNAs ( $\times 40$ dilution) 


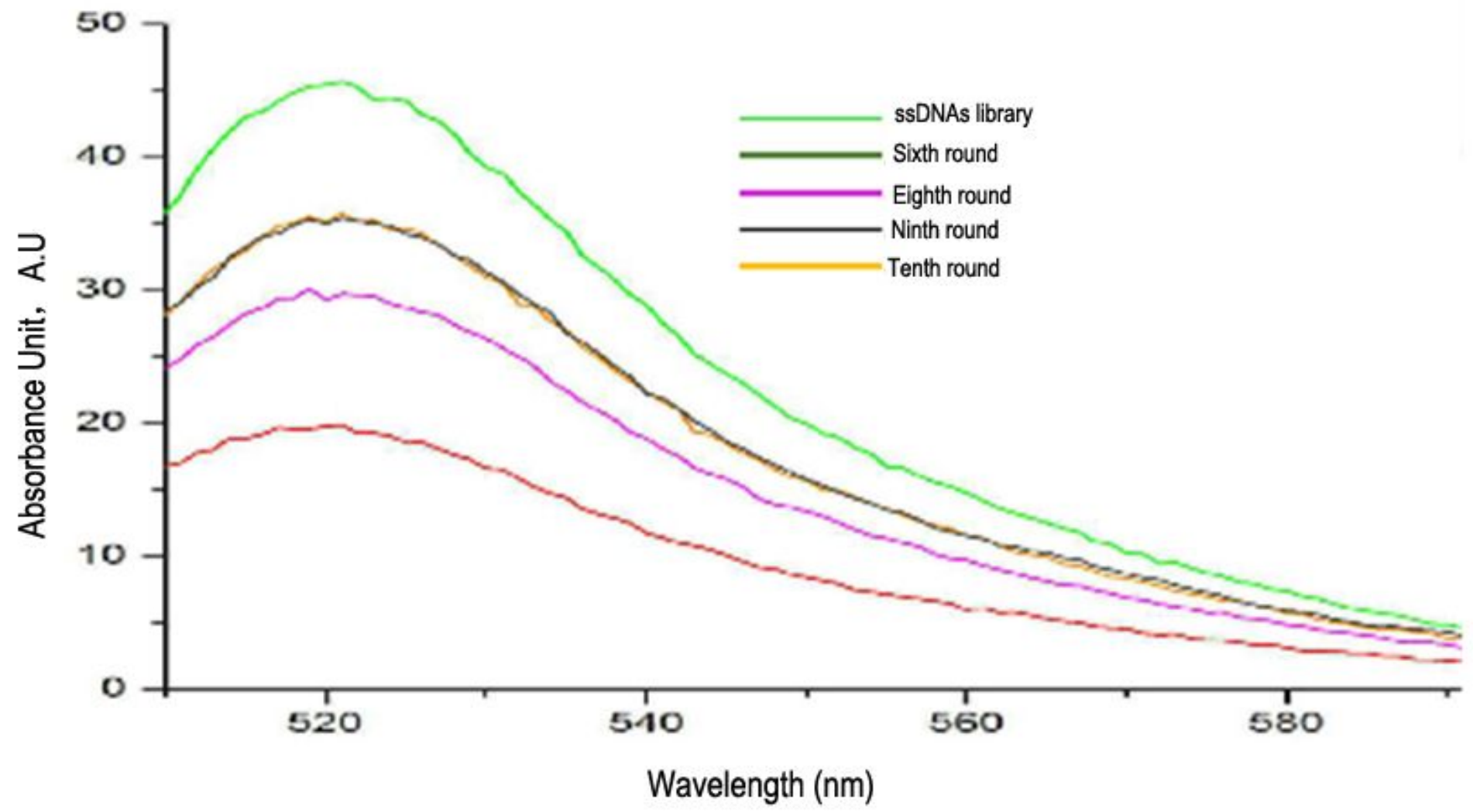

Figure 6

Fluorescence spectrum monitoring of HOS cell sub-library ssDNAs ( $\times 40$ dilution) 